\title{
Correspondence
}

Epidemiol. Infect. (2016).

doi:10.1017/S0950268816000832

\section{Adjusting for calendar time in a TND influenza study}

\section{To the Editor}

Bond and co-authors investigate, by means of simulations, how to best adjust for calendar time in a test-negative design (TND) study for influenza vaccine effectiveness (IVE) [1]. They consider three scenarios. In scenario 1 , the scenario for seasonal IVE studies, all vaccinations are administered before the epidemic. In scenarios 2 and 3, scenarios for, among others, pandemic studies, some or all of the vaccinations are administered during the epidemic. They find that for scenario 1 adjustment for calendar time is not necessary.

In the literature there is confusion concerning the need to adjust for calendar time in the TND $[2,3]$. Thanks to the investigation of Bond et al., the debate is now settled. In seasonal IVE studies it is not needed, in pandemic studies it is. The authors do not address the question why adjustment for calendar time is not needed in seasonal IVE studies. I would therefore like to add a short explanation supporting their finding: it is implied by the core assumption of the TND.

In the TND, patients seeking healthcare for an acute respiratory illness (ARI) are tested for influenza. Those who test positive for influenza are the cases, those who test negative are the controls. Vaccine effectiveness (VE) is estimated in the usual manner for case-control studies, i.e. as 1 - vaccination odds ratio (OR). The core assumption of the TND is that the incidence of non-influenza ARIs does not differ between vaccinated and unvaccinated patients [2]. In casecontrol designs adjustment for calendar time is applied if there are time dependencies that will lead to bias if ignored [4]. It has been claimed that in the TND such a time dependency is calendar time being correlated with both vaccine administration and a varying incidence of non-influenza ARIs during the epidemic [2]. This claim is incorrect. In the TND the only time dependency leading to bias in the TND is vaccine administration during the epidemic. Whether the incidence of non-influenza ARIs varies over time or not, is explained below.

Analysis of TND data is usually done by means of logistic regression. Adjustment for time is achieved by including calendar time as a covariate in the logistic model, or by conditional logistic regression stratified by week. Consider the second approach, logistic regression with matching on week. Let $A_{i}, B_{i}, C_{i}$ and $D_{i}$ be the cases and the controls enrolled in the study during the $i$ th week of the epidemic. In scenario 1 , both the vaccinated and the unvaccinated subgroups are in steady state during the epidemic. The core assumption of the TND then implies that in this scenario for the ratios of the controls the following equation applies: $C_{i} / D_{i}=N_{\text {vac. }} / N_{\text {unvac. }}=c$ (a constant), even if the incidence of non-influenza ARIs varies over time. Equivalently, for the ratios of the cases the following equation holds: $A_{i} / B_{i}=c(1-\mathrm{VE})$. It then follows that $\mathrm{OR}_{\text {adjusted }}=\mathrm{OR}_{\text {crude }}=1-\mathrm{VE}$. This explains why the crude and the adjusted estimates for scenario 1 in Table 2 of the paper by Bond et al. are almost identical. In contrast, in scenarios 2 and 3 the subgroups are not in steady state, because subjects move from the unvaccinated to the vaccinated subgroup, implying that the ratios $A_{i} / B_{i}$ and $C_{i} / D_{i}$ are not constants. However, due to the matching on week $\left(A_{i} / B_{i}\right) /\left(C_{i} / D_{i}\right)=1-\mathrm{VE}$, and thus $\mathrm{OR}_{\text {adjusted }}=1-\mathrm{VE}$, while $\mathrm{OR}_{\text {crude }} \neq 1-\mathrm{VE}$.

In summary, in a TND study seasonal IVE adjustment for calendar time is not necessary.

\section{Declaration of Interest}

None. 


\section{References}

1. Bond HS, Sullivan SG, Cowling BJ. Regression approaches in the test-negative study design for assessment of influenza vaccine effectiveness. Epidemiology and Infection. doi:10.1017/S095026881500309X. Published online: 6 January 2016.

2. Jackson ML, Nelson JC. The test negative design for estimating influenza vaccine effectiveness. Vaccine 2013; 31: $2165-2168$.

3. Jacoby P, Kelly H. Letter to the Editor. Is it necessary to adjust for calendar time in a test negative design? Responding to: Jackson ML, Nelson JC. The test negative design for estimating influenza vaccine effectiveness. Vaccine 2014; 32: 2942.

4. Vandenbroucke JP, Pearce N. Case-control studies: basic concepts. International Journal of Epidemiology 2012; 41: 1480-1489.

JOZEF J. P. NAUTA

Abbott Established Pharmaceuticals Division, Abbott Healthcare Products B.V., C.J. van Houtenlaan 36, 1381 CP Weesp, The Netherlands

(Email: jos.nauta@abbott.com)

Epidemiol. Infect. (2016).

doi:10.1017/S0950268816000959

\section{The authors reply:}

We thank Nauta [1] for providing further explanation on the need to control calendar time in TNDs. We agree that such adjustment is probably redundant in temperate climates with well-defined influenza seasons and well-defined influenza vaccination campaigns. However, we caution those researchers conducting TND studies in tropical and sub-tropical regions where there may be multiple influenza seasons. A vaccination campaign scheduled for April or September, when the Southern and Northern hemisphere vaccines are typically available, may fall before, during or even after the peak of the influenza season. When the campaign and influenza season coincide, vaccination status would not be in steady state and the need to adjust for calendar time persists.

\section{Reference}

1. Nauta JJP. Adjusting for calendar time in a TND influenza study [Letter]. Epidemiology and Infection. doi: $10.1017 / \mathrm{S} 0950268816000832$.

S. G. SULLIVAN ${ }^{1,2}$, B. J. COWLING ${ }^{3}$

${ }^{1}$ WHO Collaborating Centre for Reference and Research on Influenza, Melbourne, Australia

${ }^{2}$ Fielding School of Public Health, University of California, Los Angeles, CA, USA

${ }^{3}$ WHO Collaborating Centre for Infectious Disease Epidemiology and Control, School of Public Health, Li Ka Shing Faculty of Medicine, The University of Hong Kong,

Hong Kong Special Administrative Region, China

(Email: sheena.sullivan@influenzacentre.org) 